Probability, Networks and Algorithms

Probability, Networks and Algorithms

PNA On a generic class of Lévy-driven vacation models

O.J. Boxma, O. Kella, M.R.H. Mandjes

Report PNA-R07 10 November 2007 
Centrum voor Wiskunde en Informatica (CWI) is the national research institute for Mathematics and Computer Science. It is sponsored by the Netherlands Organisation for Scientific Research (NWO).

CWI is a founding member of ERCIM, the European Research Consortium for Informatics and Mathematics.

CWI's research has a theme-oriented structure and is grouped into four clusters. Listed below are the names of the clusters and in parentheses their acronyms.

\section{Probability, Networks and Algorithms (PNA)}

Software Engineering (SEN)

Modelling, Analysis and Simulation (MAS)

Information Systems (INS)

Copyright (C) 2007, Stichting Centrum voor Wiskunde en Informatica

P.O. Box 94079, 1090 GB Amsterdam (NL)

Kruislaan 413, 1098 SJ Amsterdam (NL)

Telephone +31205929333

Telefax +31205924199

ISSN 1386-3711 


\title{
On a generic class of Lévy-driven vacation models
}

\begin{abstract}
This paper analyzes a generic class of queueing systems with server vacation. The special feature of the models considered is that the duration of the vacations explicitly depends on the buffer content evolution during the previous active period (i.e., the time elapsed since the previous vacation). During both active periods and vacations the buffer content evolves as a Lévy process. For two specific classes of models the Laplace-Stieltjes transform of the buffer content distribution at switching epochs between successive vacations and active periods, and in steady state, is derived.
\end{abstract}

2000 Mathematics Subject Classification: 60K05, 60K25

Keywords and Phrases: Lévy process, storage process, queues with server vacations 



\title{
On a generic class of Lévy-driven vacation models
}

\author{
Onno Boxma* $\quad$ Offer Kella $^{\dagger} \quad$ Michel Mandjes ${ }^{\ddagger}$
}

\begin{abstract}
This paper analyzes a generic class of queueing systems with server vacation. The special feature of the models considered is that the duration of the vacations explicitly depends on the buffer content evolution during the previous active period (i.e., the time elapsed since the previous vacation). During both active periods and vacations the buffer content evolves as a Lévy process. For two specific classes of models the Laplace-Stieltjes transform of the buffer content distribution at switching epochs between successive vacations and active periods, and in steady state, is derived.
\end{abstract}

Keywords: Lévy process, storage process, queues with server vacations

AMS Subject Classification: Primary 60K05; Secondary 60K25

\section{Introduction}

Motivated by problems arising in the areas of computer, communication, and production systems, over the past decades substantial attention has been paid to the analysis of vacation models. These are queueing models in which the server alternates between active and passive modes; passive periods, i.e., periods in which the server does not work, are referred to as 'vacations'. It is commonly assumed that the durations of the vacations constitute a sequence of independent, identically distributed random variables, where this sequence is also assumed to be independent of the past evolution of the queueing system. For extensive surveys on this type of vacation queues we refer to [3, 4], and the book [8].

In [2] we have departed from the independence assumptions mentioned above, and explicitly model positive correlation between subsequent active and passive periods. Notice that in many situations there is such a positive correlation; an example is provided by a queue

*EURANDOM and Department of Mathematics and Computer Science, Eindhoven University of Technology, P.O. Box 513, 5600 MB Eindhoven, The Netherlands (Email: boxma@win.tue.nl).

${ }^{\dagger}$ Department of Statistics, The Hebrew University of Jerusalem, Mount Scopus, Jerusalem 91905, Israel (Email: Offer.Kella@huji.ac.il).

${ }^{\ddagger}$ Korteweg-de Vries Institute for Mathematics, The University of Amsterdam, Plantage Muidergracht 24, 1018 TV Amsterdam, The Netherlands. MM is also affiliated to CWI, P.O. Box 94079, 1090 GB Amsterdam, The Netherlands, and EuRANDOM, P.O. Box 513, 5600 MB Eindhoven, The Netherlands (Email: mmandjes@science.uva.nl). 
in a two-queue polling system, in which the intervals in which the other queue are served are identified with server vacations. In the setup of [2] it is assumed that the buffer content during both the active periods and the passive periods evolves as a Lévy process (which is assumed to have no negative jumps during the active periods, and to be a subordinator during the vacations). We refer to [2] for additional references on vacation models with some form of dependence. See also [6], where another form of correlation between active periods and vacations is considered, with special attention for the interesting phenomenon of a possible explosion of an infinite number of active periods and vacation periods in a finite time interval.

In the present paper we analyze a model similar to the one in [2], but we allow the nature of the dependence between the active and passive periods to be more general. To explain the model, we first consider the following elementary system. Focus on the start of an active period; let $\tau$ be the time until the buffer is empty, and let $T$ be an independent exponential clock. Then we sample the length of the next vacation as a random variable $S_{1}$ if $T$ is smaller than $\tau$, and as $S_{2}$ otherwise. It is clear that this model is rather flexible, as it allows for a broad range of possible correlations (both positive and negative) between the active and passive periods.

In the paper we study two generalizations of the above model. In the first, which we will refer to as Model 1 and which is treated in Section 2, we have $d$ exponential clocks (whose means are not necessarily identical). If the set of clocks that is still active at time $\tau$ is $A \subseteq$ $\{1, \ldots, d\}$, then the duration of the next vacation is distributed as a random variable $S_{A}$. In Model 2, which is addressed in Section 3, there is just one clock, but this clock has a phasetype distribution. The distribution of the next vacation then depends on whether the clock is still active at $\tau$ or not.

For both models we compute the Laplace-Stieltjes transform of the steady-state storage level. We do so by first analyzing the storage level at 'switch epochs', that is, epochs at which there is a transition from the passive to the active mode. Then we use an 'averaging procedure' to translate the results for the embedded epochs into time-average results. We are also able to explicitly characterize the correlation between subsequent active and passive periods.

\section{Model 1: vacation determined by multiple exponential clocks}

Consider the following model of a storage system that alternates between service periods (or: active periods) and vacations (or: passive periods). Its dynamics are described as follows.

- Suppose the storage level is $z \geq 0$ at the beginning of a service period. Then the storage level evolves as a Lévy process $X(\cdot)$ (which is assumed to have no negative jumps). It has a drift downwards, that is, $\varrho_{X}:=-\mathbb{E}[X(1)]$ is positive (and finite). Observe that 
the process will actually attain level 0 , say at time $\tau \equiv \tau(z)$ :

$$
\tau(z):=\inf \{t \geq 0: z+X(t)=0\} .
$$

Let us introduce some additional notation. The Laplace exponent of $X(\cdot)$ is given by $\varphi(\alpha):=\log \mathbb{E}[\exp (-\alpha X(1))]$, and hence $\mathbb{E}[\exp (-\alpha X(t))]=e^{\varphi(\alpha) t}$ and $\varrho_{X}=\varphi^{\prime}(0)$. Also, $\psi(\cdot)$ denotes the inverse of $\varphi(\cdot)$. It is well-known that for any Lévy process that has no negative jumps, $\tau(\cdot)$ is a Lévy process itself, with Laplace exponent $-\psi(\alpha)$, that is

$$
\mathbb{E}\left[e^{-\alpha \tau(z)}\right]=e^{-\psi(\alpha) z},
$$

see, for instance, Thm. 46.3 in [7]. Notice that, for $\alpha \geq 0, \psi(\alpha)$ is uniquely defined as the inverse of $\varphi(\cdot)$, as $\varphi(\cdot)$ increases on $[0, \infty)$.

- At the beginning of the service period, we initiate $d \in \mathbb{N}$ independent exponential clocks, say, $T_{1}, \ldots, T_{d}$; define $\mathscr{N}_{d}:=\{1, \ldots, d\}$. Let $A(t)$ be the set of clocks which are still active at time $t \geq 0$ :

$$
A(t):=\left\{i \in \mathscr{N}_{d}: T_{i}>t\right\}
$$

For any $A \subseteq \mathscr{N}_{d}$ we define a nonnegative random variable $S_{A}$ with Laplace-Stieltjes transform (LST)

$$
\beta_{A}(\alpha):=\mathbb{E}\left[e^{-\alpha S_{A}}\right]
$$

with mean $\mu_{A}=-\beta_{A}^{\prime}(0)<\infty$. To ensure a nontrivial model, it is assumed that $\mu_{\mathscr{N}_{d}}>0$, though it could be the case that $\mu_{A}=0$ for strict subsets $A$ of $\mathscr{N}_{d}$.

If, at the end of the service period the set of active clocks is $A$, i.e., $A(\tau(z))=A$, then the next vacation is distributed as $S_{A}$. During this vacation, traffic is generated according to a nondecreasing Lévy process (i.e., a subordinator) $Y(\cdot)$ with Laplace exponent $-\eta(\alpha):=\log \mathbb{E}[\exp (-\alpha Y(1))]$. The corresponding (positive) drift is denoted by $\varrho_{Y}:=\eta^{\prime}(0)=\mathbb{E}[Y(1)]<\infty$.

In the sequel, we refer to a consecutive active period and passive period as a 'cycle'. Let $Z_{n}$ be the storage level at the end of the $n$-th cycle. It is straightforward to derive that after the first cycle the storage level has LST (in the sequel, $\mathbb{E}_{z}$ and $\mathbb{P}_{z}$ denote means and probabilities conditional on the process starting in $z$ at time 0 ):

$$
\mathbb{E}_{z}\left[e^{-\alpha Z_{1}}\right]=\sum_{A \subseteq \mathscr{N}_{d}} \mathbb{P}_{z}(A(\tau)=A) \beta_{A}(\eta(\alpha)) .
$$

- Then a new service period starts (i.e., the Lévy process $X(\cdot)$ becomes active again), etc. 
Remark 1. A special case of the above model results by taking $X(\cdot)$ a compound Poisson process minus a positive drift, and $Y(\cdot)$ a (e.g., the same) compound Poisson process but without the drift. This is an $M / G / 1$ queue with vacations, the length of a vacation depending on the length of the preceding busy period.

Now that we have defined the dynamics of the process, we subsequently (i) indicate how the probabilities $p_{z}(A):=\mathbb{P}_{z}(A(\tau)=A)$, for $z \geq 0, A \subseteq \mathscr{N}_{d}$, can be computed; (ii) determine the steady-state distribution of the storage level at the 'switching epochs', i.e., epochs that the process switches from passive periods to active periods; (iii) study the correlation between consecutive active and passive periods; (iv) determine the steady-state distribution of the storage level in continuous time.

(i) Reflections on the computation of the $p_{z}(A)$. We first observe that $\mathbb{P}_{0}(\tau=0)=1$ so that $p_{0}\left(\mathscr{N}_{d}\right)=1$, and consequently $p_{0}(A)=0$ for all strict subsets $A$ of $\mathscr{N}_{d}$. Now consider the case $z>0$. Then

$$
p_{z}(A)=\mathbb{E}_{z}\left[\prod_{i \in A} 1_{\left\{\tau<T_{i}\right\}} \prod_{j \notin A} 1_{\left\{\tau \geq T_{j}\right\}}\right]=\mathbb{E}_{z}\left[\prod_{i \in A} e^{-\lambda_{i} \tau} \prod_{j \notin A}\left(1-e^{-\lambda_{j} \tau}\right)\right],
$$

where an empty product is one. For conciseness, we introduce for $A \subseteq \mathscr{N}_{d}$, the 'aggregate rate $^{\prime} \Lambda(A):=\sum_{i \in A} \lambda_{i}$, where an empty sum is zero. Observe that

$$
\begin{aligned}
\prod_{i \in A} e^{-\lambda_{i} \tau} \prod_{j \notin A}\left(1-e^{-\lambda_{j} \tau}\right) & =\sum_{k=0}^{n-|A|}(-1)^{k} \sum_{\{B: B \supseteq A \text { and }|B|=|A|+k\}} e^{-\Lambda(B) \tau} \\
& =\sum_{\{B: B \supseteq A\}}(-1)^{|B|-|A|} e^{-\Lambda(B) \tau} .
\end{aligned}
$$

Taking the expected values in (7), in conjunction with (2), now gives that

$$
p_{z}(A)=\sum_{\{B: B \supseteq A\}}(-1)^{|B|-|A|} e^{-\psi(\Lambda(B)) z} .
$$

In particular

$$
p_{z}\left(\mathscr{N}_{d}\right)=e^{-\psi\left(\Lambda\left(\mathscr{N}_{d}\right)\right) z} .
$$

(As an aside, we mention that it is easy to check that (8) and (9) remain valid for $z=0$. In particular, for $A$ being a strict subset of $\mathscr{N}_{d}$, and with $A^{c}:=\mathscr{N}_{d} \backslash A$,

$$
p_{0}(A)=\sum_{\{B: B \supseteq A\}}(-1)^{|B|-|A|}=\sum_{\left\{B^{\prime}: B^{\prime} \subseteq A^{c}\right\}}(-1)^{\left|B^{\prime}\right|}=0,
$$

as the number of subsets of $A^{c}$ is $2^{\left|A^{c}\right|}$, where half of them are odd and the other half even. Also, evidently, (9) is in agreement with $p_{0}\left(\mathscr{N}_{d}\right)=1$.) 
(ii) Steady-state distribution of the storage level at the switching epochs. We now consider the steady-state distribution of the Markov process $\left(Z_{n}\right)_{n \in \mathbb{N}}$. Clearly, such a stationary distribution, say $Z$, exists (independent of the initial state $Z_{0}$ ): it is evident that the process is regenerative, and the mean regeneration time is finite.

Denote by $\zeta(\cdot)$ the LST of the steady-state distribution $Z$ of $\left(Z_{n}\right)_{n \in \mathbb{N}}$. Then, from (5) and (8), for $\alpha \geq 0$,

$$
\begin{aligned}
\zeta(\alpha) & =\sum_{A \subseteq B \subseteq \mathscr{N}_{d}}(-1)^{|B|-|A|} \zeta(\psi(\Lambda(B))) \beta_{A}(\eta(\alpha)) \\
& =\beta_{\varnothing}(\eta(\alpha))+\sum_{\substack{B \subseteq \mathcal{N}_{d} \\
B \neq \varnothing}}\left(\sum_{A \subseteq B}(-1)^{|A|} \beta_{A}(\eta(\alpha))\right)(-1)^{|B|} \zeta(\psi(\Lambda(B))) .
\end{aligned}
$$

Later we will use the identity that is obtained after inserting $\alpha=0$; we then find

$$
0=\sum_{\substack{B \subseteq \mathcal{M}_{d} \\ B \neq \varnothing}}\left(\sum_{A \subseteq B}(-1)^{|A|}\right)(-1)^{|B|} \zeta(\psi(\Lambda(B))) .
$$

We remark that the last equation is also obvious from the fact that, for any nonempty $B$, $\sum_{A \subseteq B}(-1)^{|A|}=0$, cf. (10).

From (11) we observe that if we know, for any $B \subseteq \mathscr{N}_{d}$, the value of $\zeta(\psi(\Lambda(B)))$, then we have identified the LST $\zeta(\cdot)$ of $Z$. These constants can be found as follows. For each $B^{\prime} \subseteq \mathscr{N}_{d}$ we substitute $\alpha:=\psi\left(\Lambda\left(B^{\prime}\right)\right)$. In this way one obtains a system of linear equations defining the missing constants. We note that it is possible that for two or more different sets $B^{\prime}$ the values of $\Lambda\left(B^{\prime}\right)$ are the same. In this case the number of unknowns is reduced, but the number of equations is reduced accordingly.

Example. Consider the simplest case, i.e., $d=1$. Then the above formulae yield that

$$
\zeta(\alpha)=\beta_{\varnothing}(\eta(\alpha))-\left[\beta_{\varnothing}(\eta(\alpha))-\beta_{\{1\}}(\eta(\alpha))\right] \zeta\left(\psi\left(\lambda_{1}\right)\right)
$$

so that

$$
\zeta\left(\psi\left(\lambda_{1}\right)\right)=\frac{\beta_{\varnothing}\left(\eta\left(\psi\left(\lambda_{1}\right)\right)\right)}{\beta_{\varnothing}\left(\eta\left(\psi\left(\lambda_{1}\right)\right)\right)+1-\beta_{\{1\}}\left(\eta\left(\psi\left(\lambda_{1}\right)\right)\right)}
$$

and thus

$$
\zeta(\alpha)=\frac{\beta_{\varnothing}\left(\eta\left(\psi\left(\lambda_{1}\right)\right)\right) \beta_{\{1\}}(\eta(\alpha))+\left(1-\beta_{\{1\}}\left(\eta\left(\psi\left(\lambda_{1}\right)\right)\right)\right) \beta_{\varnothing}(\eta(\alpha))}{\beta_{\varnothing}\left(\eta\left(\psi\left(\lambda_{1}\right)\right)\right)+1-\beta_{\{1\}}\left(\eta\left(\psi\left(\lambda_{1}\right)\right)\right)} .
$$

When $\mu_{\varnothing}=0$, and thus $\beta_{\varnothing}(\cdot)=1$, we have

$$
\zeta(\alpha)=\frac{\beta_{\{1\}}(\eta(\alpha))+\left(1-\beta_{\{1\}}\left(\eta\left(\psi\left(\lambda_{1}\right)\right)\right)\right)}{2-\beta_{\{1\}}\left(\eta\left(\psi\left(\lambda_{1}\right)\right)\right)} .
$$


(iii) Correlation between consecutive active and passive periods. We note that, due to the standard identity $\operatorname{Cov}(U, V)=\operatorname{Cov}(U, \mathbb{E}[V \mid U])$, for any given initial level $z \geq 0$, the covariance between the durations of the active and passive periods is

$$
\operatorname{Cov}_{z}\left(\tau, \sum_{A \subseteq \mathscr{N}_{d}} 1_{\{A(\tau)=A\}} \mu_{A}\right)=\sum_{A \subseteq \mathscr{N}_{d}} \mu_{A} \operatorname{Cov}_{z}\left(\tau, 1_{\{A(\tau)=A\}}\right) .
$$

This covariance can be computed in a rather straightforward manner. To this end, first observe that it follows immediately from (2) that

$$
\mathbb{E}_{z}\left[\tau e^{-\lambda \tau}\right]=-\frac{\mathrm{d}}{\mathrm{d} \lambda} \mathbb{E}_{z}\left[e^{-\lambda \tau}\right]=z \psi^{\prime}(\lambda) e^{-\psi(\lambda) z}
$$

and as $\mathbb{E}_{z}[\tau]=z / \varrho_{X}=z \psi^{\prime}(0)$ we have that

$$
\operatorname{Cov}\left(\tau, e^{-\lambda \tau}\right)=-z e^{-\psi(\lambda) z}\left(\psi^{\prime}(0)-\psi^{\prime}(\lambda)\right)
$$

which is strictly negative when $X(\cdot)$ is not a linear drift (as $\varphi(\cdot)$ is then strictly convex and thus $\psi(\cdot)$ is strictly concave); the covariance is zero when $X(\cdot)$ is a linear drift.

Relying on (7) and (19), we can now further determine the terms in the sum in the right-hand side of (17). It is readily checked that

$$
\begin{aligned}
& \operatorname{Cov}_{z}\left(\tau, 1_{\{A(\tau)=A\}}\right)=\sum_{\{B: B \supseteq A\}}(-1)^{|B|-|A|} \operatorname{Cov}_{z}\left(\tau, e^{-\Lambda(B) \tau}\right) \\
& =-z \sum_{\{B: B \supseteq A\}}(-1)^{|B|-|A|} e^{-\psi(\Lambda(B)) z}\left(\psi^{\prime}(0)-\psi^{\prime}(\Lambda(B))\right)
\end{aligned}
$$

and thus the covariance (17) between the active period and the next passive period is given by

$$
\begin{aligned}
& -z \sum_{A \subseteq B \subseteq \mathscr{N}_{d}} \mu_{A} \cdot(-1)^{|B|-|A|} \cdot e^{-\psi(\Lambda(B)) z} \cdot\left(\psi^{\prime}(0)-\psi^{\prime}(\Lambda(B))\right) \\
& =-z \sum_{B \subseteq \mathscr{N}_{d}}\left(\sum_{A \subseteq B}(-1)^{|A|} \mu_{A}\right)(-1)^{|B|} e^{-\psi(\Lambda(B)) z} \cdot\left(\psi^{\prime}(0)-\psi^{\prime}(\Lambda(B))\right) \\
& =-z \sum_{\substack{B \subseteq \mathcal{N}_{d} \\
B \neq \varnothing}}\left(\sum_{A \subseteq B}(-1)^{|A|} \mu_{A}\right)(-1)^{|B|} e^{-\psi(\Lambda(B)) z} \cdot\left(\psi^{\prime}(0)-\psi^{\prime}(\Lambda(B))\right) .
\end{aligned}
$$

This expression reveals that if, for each $B, \sum_{A \subset B}(-1)^{|A|} \mu_{A}$ is positive when $|B|$ is even (odd) and negative when $|B|$ is odd (even), then the covariance will be negative (positive, respectively). 
For example, in case $d=1$ we have that for $B=\{1\}, \sum_{A \subset B}(-1)^{|A|} \mu_{A}=\mu_{\varnothing}-\mu_{\{1\}}$. In this particular case the covariance is

$$
-z\left(\mu_{\varnothing}-\mu_{\{1\}}\right) e^{-\psi\left(\lambda_{1}\right) z}\left(\psi^{\prime}(0)-\psi^{\prime}\left(\lambda_{1}\right)\right)
$$

(iv) Steady-state distribution of the storage level in continuous time. In order to compute the stationary distribution for the continuous time process we look at a single cycle which begins with the stationary distribution of the embedded Markov process $\left(Z_{n}\right)_{n \in \mathbb{N}}$. Then

$$
\begin{aligned}
& \mathbb{E}\left[\int_{0}^{\tau} e^{-\alpha X(t)} \mathrm{d} t\right]=\int_{0}^{\infty} \mathbb{E}\left[\int_{0}^{\tau(z)} e^{-\alpha X(t)} \mathrm{d} t\right] \mathrm{d} \mathbb{P}(Z \leq z) \\
& =\int_{0}^{\infty} \mathbb{E}\left[\int_{0}^{\tau(z)} e^{-\varphi(\alpha) t} \mathrm{~d} t\right] \mathrm{d} \mathbb{P}(Z \leq z)=\int_{0}^{\infty} \mathbb{E}\left[\frac{1-e^{-\varphi(\alpha) \tau(z)}}{\varphi(\alpha)}\right] \mathrm{d} \mathbb{P}(Z \leq z)= \\
& =\int_{0}^{\infty}\left(\frac{1-e^{-\alpha z}}{\varphi(\alpha)}\right) \mathrm{d} \mathbb{P}(Z \leq z)=\frac{1-\zeta(\alpha)}{\varphi(\alpha)} .
\end{aligned}
$$

In the last line, we have used (2) and $\psi(\varphi(\alpha))=\alpha$.

With $V$ denoting a random variable distributed like a passive period, we have

$$
\mathbb{E}\left[\int_{0}^{V} e^{-\alpha Y(t)} \mathrm{d} t\right]=\mathbb{E}\left[\int_{0}^{V} e^{-\eta(\alpha) t} \mathrm{~d} t\right]=\frac{1-\mathbb{E}\left[e^{-\eta(\alpha) V}\right]}{\eta(\alpha)}=\frac{1-\mathbb{E}\left[e^{-\alpha Y(V)}\right]}{\eta(\alpha)} .
$$

Since $Z_{1}$ is distributed like $Y(V)$, and using that the process $\left(Z_{n}\right)_{n \in \mathbb{N}}$ is stationary, it immediately follows that

$$
\mathbb{E}\left[\int_{0}^{V} e^{-\alpha Y(t)} \mathrm{d} t\right]=\frac{1-\zeta(\alpha)}{\eta(\alpha)} .
$$

Conclude that the time-stationary distribution of the process (that is, in continuous time) is given by

$$
\frac{\mathbb{E}\left[\int_{0}^{\tau} e^{-\alpha X(t)} \mathrm{d} t\right]+\mathbb{E}\left[\int_{0}^{V} e^{-\alpha Y(t)} \mathrm{d} t\right]}{\mathbb{E}[\tau]+\mathbb{E}[V]}=\frac{(1-\zeta(\alpha))\left(\frac{1}{\varphi(\alpha)}+\frac{1}{\eta(\alpha)}\right)}{-\zeta^{\prime}(0)\left(\frac{1}{\varphi^{\prime}(0)}+\frac{1}{\eta^{\prime}(0)}\right)} .
$$

Using earlier results this expression can be further simplified, as follows. Denoting $\beta_{A}^{e}(\alpha):=$ $\left(1-\beta_{A}(\alpha)\right) /\left(\mu_{A} \alpha\right)$ when $\mu_{A}>0$ and $\beta_{A}^{e}(\alpha):=1$ when $\mu_{A}=0$, and setting

$$
c_{A}:=\mu_{A} \sum_{\left\{B: A \subseteq B \subseteq \mathscr{N}_{d}\right\}}(-1)^{|B|-|A|} \zeta(\psi(\Lambda(B)))=\mu_{A} \cdot p_{Z}(A),
$$

we have from (11) and (12) that

$$
\frac{1-\zeta(\alpha)}{-\zeta^{\prime}(0)}=\frac{\eta(\alpha) \sum_{A \subseteq \mathscr{N}_{d}} c_{A} \beta_{A}^{e}(\eta(\alpha))}{\eta^{\prime}(0) \sum_{A \subseteq \mathscr{N}_{d}} c_{A}} .
$$


This entails that (26) simplifies to

$$
\frac{\sum_{A \subseteq \mathscr{N}_{d}} c_{A} \beta_{A}^{e}(\eta(\alpha))}{\sum_{A \subseteq \mathscr{N}_{d}} c_{A}} \cdot \frac{1+\frac{\eta(\alpha)}{\varphi(\alpha)}}{1+\frac{\eta^{\prime}(0)}{\varphi^{\prime}(0)}} .
$$

Remark 2. It can be easily checked that for $d=1$ the resulting LST is

$$
\frac{\mu_{\{1\}} \beta_{\varnothing}\left(\eta\left(\psi\left(\lambda_{1}\right)\right)\right) \beta_{\{1\}}^{e}(\eta(\alpha))+\mu_{\varnothing}\left(1-\beta_{\{1\}}\left(\eta\left(\psi\left(\lambda_{1}\right)\right)\right)\right) \beta_{\varnothing}^{e}(\eta(\alpha))}{\mu_{\{1\}} \beta_{\varnothing}\left(\eta\left(\psi\left(\lambda_{1}\right)\right)\right)+\mu_{\varnothing}\left(1-\beta_{\{1\}}\left(\eta\left(\psi\left(\lambda_{1}\right)\right)\right)\right)} \cdot \frac{1+\frac{\eta(\alpha)}{\varphi(\alpha)}}{1+\frac{\eta^{\prime}(0)}{\varphi^{\prime}(0)}} .
$$

If we assume in addition that $\mu_{\varnothing}=0$ (that is, there is no vacation when the active period is too long) then this LST further simplifies to

$$
\beta_{\{1\}}^{e}(\eta(\alpha)) \cdot \frac{1+\frac{\eta(\alpha)}{\varphi(\alpha)}}{1+\frac{\eta^{\prime}(0)}{\varphi^{\prime}(0)}} .
$$

At first sight, it may seem that this is a mistake as it does not depend on $\lambda_{1}$. However, some thought reveals that if $\tau \geq T_{1}$ then there is no vacation. This means that during the next cycle the process starts from zero and hence the hitting time of zero is zero, and thus is majorized by the next exponential, and as a consequence a vacation (with LST $\beta_{\{1\}}(\cdot)$ ) will begin. Therefore, the process is identical to one where no comparison with exponentials is made, and in which we have just active periods followed by independent vacations with LST $\beta_{\{1\}}(\cdot)$. A related model was considered in [5]. The same phenomenon will occur if $\mu_{A}=0$ for all $A$ that are strict subsets of $\mathscr{N}_{d}$, and $\mu_{\mathscr{N}_{d}}>0$.

Remark 3. A special case of the model with $d=1$ is the following. Assume that the length of a cycle is $\max \left(\tau, T_{\lambda}\right)$ where $T_{\lambda} \sim \exp (\lambda)$ and is independent of all other stochastic quantities involved in the system. If $\tau<T_{\lambda}$, then the length of the vacation is $T_{\lambda}-\tau$ and otherwise it is zero. This model is indeed equivalent to the model above with the choices $d=1, \lambda_{1}=\lambda$, $\mathbb{P}\left(S_{\varnothing} \leq t\right)=1_{[0, \infty)}(t)$ and $\mathbb{P}\left(S_{\{1\}} \leq t\right)=\left(1-e^{-\lambda t}\right) 1_{[0, \infty)}(t)$ (that is, with $\beta_{\varnothing}(\alpha)=1$ and $\left.\beta_{\{1\}}(\alpha)=\beta_{\{1\}}^{e}(\alpha)=\lambda /(\lambda+\alpha)\right)$.

Remark 4. When $\varphi(\alpha)=r \alpha-\eta(\alpha)$, that is when during active periods there is a deterministic outflow at rate $r$ while during vacations there is none, the LST of the last term in (29) is given by

$$
\frac{1+\frac{\eta(\alpha)}{\varphi(\alpha)}}{1+\frac{\eta^{\prime}(0)}{\varphi^{\prime}(0)}}=\frac{\frac{r \alpha}{\varphi(\alpha)}}{\frac{r}{\varphi^{\prime}(0)}}=\frac{\alpha \varphi^{\prime}(0)}{\varphi(\alpha)}
$$


which is simply the LST of the steady-state storage level of a reflected Lévy process with exponent $\varphi$ [1, Ch. IX]. This leads to the decomposition result discovered in [5] for a model in which the length of the vacation is independent of the previous active period, but on the other hand is a general stopping time with respect to some filtration (not necessarily the one generated by $Y(\cdot))$.

Remark 5. Let $F_{A}(\cdot):=\mathbb{P}\left(S_{A} \leq \cdot\right)$. Whenever a vacation is zero it is as if we are immediately starting another vacation with distribution

$$
\frac{F_{\mathscr{N}_{d}}(t)-F_{\mathscr{N}_{d}}(0)}{1-F_{\mathscr{N}_{d}}(0)} \text {. }
$$

Therefore without loss of generality we may assume that $F_{A}(0)=0$ and, in particular, that $\mu_{A}>0$ for all $A$. For example, if $F_{A}(0)>0$, then when replacing $F_{A}(t)$ with

$$
G_{A}(t):=F_{A}(t)-F_{A}(0)+F_{A}(0) \frac{F_{\mathscr{N}_{d}}(t)-F_{\mathscr{N}_{d}}(0)}{1-F_{\mathscr{N}_{d}}(0)}
$$

the continuous-time process remains the same, but $G_{A}(0)=0$ (it evidently has impact on the embedded process $\left(Z_{n}\right)_{n \in \mathbb{N}}$, though).

\section{Model 2: vacation determined by a phase-type clock}

This model is similar to the one of the previous section, in that the storage level is determined by alternating active and passive periods. Also, as before, the buffer level at the beginning of the active period has impact on the duration of the vacation. Suppose at the beginning of the active period the buffer level was $z$, and let $\tau$ be the time it takes the system to empty. Now consider a 'clock' random variable $T$ which has a phase-type distribution. If $T \leq \tau$ the next vacation period lasts for a random time with LST $\beta_{1}(\cdot)$, and if $T>\tau$ it lasts for a random time with LST $\beta_{2}(\cdot)$. Define $p_{z}(1):=\mathbb{P}_{z}(T \leq \tau)$ and $p_{z}(2):=\mathbb{P}_{z}(T>\tau)$. Similar to the previous section, we first (i) compute the probabilities $p_{z}(1)$ and $p_{z}(2)$, then (ii) determine the storagelevel distribution at 'switch epochs' (i.e., as before, epochs at which the active period starts), then (iii) analyze the correlation between subsequent active and passive periods, and finally (iv) determine the steady-state distribution at an arbitrary point in time.

(i) Reflections on the computation of the $p_{z}(i)$, for $i=1,2$. Any phase-type distribution is characterized by a $d$-dimensional $(d \in \mathbb{N})$ vector $\gamma$, and a $d \times d$ matrix $Q$. Here $\gamma$ is the 'initial distribution', and $Q$ is such that there is a generator matrix $\bar{Q}$ that can be written as

$$
\bar{Q}=\left(\begin{array}{cc}
Q & q \\
\overrightarrow{0}^{\mathrm{T}} & 0
\end{array}\right)
$$

with $q$ chosen such that the rows sum to 0 (here $\overrightarrow{0}$ is a $d$-dimensional vector with all its entries equal to 1 , and the superscript ' $\mathrm{T}$ ' denotes transpose). The last row (containing just 
zeroes) indicates that state $d+1$ is absorbing; notice also that the vector $q$ is necessarily componentwise nonnegative. From [1, Prop. III.4.1] we immediately have that

$$
\mathbb{P}(T \geq t)=\gamma e^{Q t} \overrightarrow{1}
$$

Let $\lambda_{1}, \ldots, \lambda_{d}$ denote the eigenvalues of $Q$, and let $\Lambda:=\operatorname{diag}\left\{\lambda_{1}, \ldots, \lambda_{d}\right\}$; all eigenvalues have a negative real part [1, Section II.4d]. Assume for the moment that all eigenvalues of $Q$ are simple, so that we have, for some matrix $S$,

$$
\begin{aligned}
& p_{z}(2)=\int_{0}^{\infty} \mathbb{P}(T>t) \mathrm{d} \mathbb{P}_{z}(\tau \leq t)=\int_{0}^{\infty} \gamma e^{Q t} \overrightarrow{1} \mathrm{~d} \mathbb{P}_{z}(\tau \leq t)= \\
& \int_{0}^{\infty} \gamma S e^{\Lambda t} S^{-1} \overrightarrow{1} \mathrm{~d} \mathbb{P}_{z}(\tau \leq t)=\sum_{i=1}^{d} \delta_{i} e^{-\psi\left(\lambda_{i}\right) z}
\end{aligned}
$$

for suitable coefficients $\delta_{1}, \ldots, \delta_{d}$. The last equality follows by combining the fact that $\gamma S \mathrm{e}^{\Lambda t} S^{-1} \overrightarrow{1}$ is some weighted sum of terms $\mathrm{e}^{-\lambda_{i} t}$ with Formula (2) (as an aside we remark that if $\lambda_{i}$ and $\lambda_{j}$ are complex conjugates, then so are $e^{-\psi\left(\lambda_{i}\right) z}$ and $e^{-\psi\left(\lambda_{j}\right) z}$ ). In many important cases, however, the eigenvalues of $Q$ are not simple. If for instance $T$ is $\operatorname{Erlang}(d, \lambda)$ distributed, it is easily verified that the eigenvalue $\lambda$ has multiplicity $d$. In general one could say that for any $T$ having a phase-type distribution there are constants $\delta_{i j}$ such that

$$
\mathbb{P}(T>t)=\sum_{i=1}^{k} \sum_{j=1}^{m_{i}} \delta_{i j} t^{j-1} e^{-\lambda_{i} t}
$$

here the $\lambda_{i}$ are, as before, the eigenvalues of $Q, k \in\{1, \ldots, d\}$ is the number of distinct eigenvalues, and $m_{i}$ is the multiplicity of the $i$ th eigenvalue (so that $\sum_{i=1}^{k} m_{i}=d$ ). It is now readily verified that

$$
p_{z}(2)=\mathbb{P}_{z}(T>\tau)=\sum_{i=1}^{k} \sum_{j=1}^{m_{i}} \delta_{i j} \mathbb{E}_{z}\left[\tau^{j-1} e^{-\lambda_{i} \tau}\right],
$$

which can be rewritten as

$$
\sum_{i=1}^{k} \sum_{j=1}^{m_{i}} \delta_{i j}(-1)^{j-1}\left(\left.\frac{\mathrm{d}^{j-1}}{\mathrm{~d} \lambda^{j-1}} e^{-\psi(\lambda) z}\right|_{\lambda=\lambda_{i}}\right) .
$$

It now follows that we can identify coefficients $\bar{\delta}_{i j}$ such that

$$
p_{z}(2)=\sum_{i=1}^{k} \sum_{j=1}^{m_{i}} \bar{\delta}_{i j} z^{j-1} e^{-\psi\left(\lambda_{i}\right) z} .
$$

Example. Consider the case that $T$ has an $\operatorname{Erlang}(2, \lambda)$ distribution; we have $k=1, m_{1}=2$, and $\lambda_{1}=\lambda$. It is straightforward to obtain that $p_{z}(2)=e^{-\psi(\lambda) z}\left(1+\lambda \psi^{\prime}(\lambda) z\right)$. In other words, in this case we have that $\bar{\delta}_{11}=1$ and $\bar{\delta}_{12}=\lambda \psi^{\prime}(\lambda)$. 
(ii) Steady-state distribution of the storage level at the switching epochs. Let $Z$ denote the steady-state storage level at switching epochs. We immediately find the following relation for the $\operatorname{LST} \zeta(\cdot)$ of $Z$ :

$$
\zeta(\alpha)=\mathbb{E}\left[p_{Z}(1)\right] \beta_{1}(\eta(\alpha))+\mathbb{E}\left[p_{Z}(2)\right] \beta_{2}(\eta(\alpha))
$$

Using the expressions for $p_{z}(i)$ derived above, we find

$$
\begin{gathered}
\zeta(\alpha)=\mathbb{E}\left[1-\sum_{i=1}^{k} \sum_{j=1}^{m_{i}} \bar{\delta}_{i j} Z^{j-1} e^{-\psi\left(\lambda_{i}\right) Z}\right] \beta_{1}(\eta(\alpha))+\mathbb{E}\left[\sum_{i=1}^{k} \sum_{j=1}^{m_{i}} \bar{\delta}_{i j} Z^{j-1} e^{-\psi\left(\lambda_{i}\right) Z}\right] \beta_{2}(\eta(\alpha)) \\
=\left(1-\sum_{i=1}^{k} \sum_{j=1}^{m_{i}} \bar{\delta}_{i j}(-1)^{j-1} \zeta^{(j-1)}\left(\psi\left(\lambda_{i}\right)\right)\right) \beta_{1}(\eta(\alpha))+ \\
\left(\sum_{i=1}^{k} \sum_{j=1}^{m_{i}} \bar{\delta}_{i j}(-1)^{j-1} \zeta^{(j-1)}\left(\psi\left(\lambda_{i}\right)\right)\right) \beta_{2}(\eta(\alpha)) .
\end{gathered}
$$

The $d$ constants $\zeta^{(j-1)}\left(\psi\left(\lambda_{i}\right)\right)$ can be identified by inserting $\alpha=\psi\left(\lambda_{i}\right)$ (for $i=1, \ldots, k$ ) into the $(j-1)$ th derivative of the above equation (for $j=1$ to $m_{i}$ ).

(iii) Correlation between consecutive active and passive periods. The covariance between active and passive periods is, with $\mu_{i}:=-\beta_{i}^{\prime}(0)$, for an initial level $z \geq 0$, given by

$$
\mu_{1} \operatorname{Cov}_{z}\left(\tau, 1_{\{T \leq \tau\}}\right)+\mu_{2} \operatorname{Cov}_{z}\left(\tau, 1_{\{T>\tau\}}\right) .
$$

Elementary algebra, and relying on the results for $p_{z}(i)$, yields that this equals

$$
\begin{aligned}
& \left(\mu_{1}-\mu_{2}\right) \sum_{i=1}^{k} \sum_{j=1}^{m_{i}} \delta_{i j} \operatorname{Cov}_{z}\left(\tau, \tau^{j-1} e^{-\lambda_{i} \tau}\right) \\
& =\left(\mu_{1}-\mu_{2}\right) \sum_{i=1}^{k} \sum_{j=1}^{m_{i}} \delta_{i j}\left(\mathbb{E}_{z}\left[\tau^{j} e^{-\lambda_{i} \tau}\right]-\mathbb{E}_{z}[\tau] \mathbb{E}_{z}\left[\tau^{j-1} e^{-\lambda_{i} \tau}\right]\right) .
\end{aligned}
$$

The latter expression can be further evaluated by differentiating $\mathbb{E}_{z}\left[e^{-\lambda_{i} \tau}\right]=e^{-\psi\left(\lambda_{i}\right) z}$.

(iv) Steady-state distribution of the storage level in continuous time. This can be done by mimicking the arguments used for Model 1; in particular Equations (23)-(26) remain valid.

Remark 6. It is clear that we can combine the models of this and the previous section; then there are $d$ clocks, each having a phase-type distribution, and the vacation has distribution $S_{A}$ if the set of clocks still active at $\tau$ is $A$. It is easily seen that the probabilities $p_{z}(A)$ are mixtures of terms of the type $z^{j} e^{-\beta z}$, for $j \in\{0,1, \ldots\}$ and $\beta>0$, and therefore the analysis of the previous sections carries over to this more general model. 


\section{References}

[1] S. Asmussen (2003). Applied Probability and Queues. Springer, New York.

[2] O.J. Boxma, M. Mandjes, and O. Kella (2007). On a queueing model with service interruptions. To appear in Probability in the Engineering and Informational Sciences.

[3] B.T. Doshi (1986). Queueing systems with vacations - a survey. Queueing Systems 1, 29-66.

[4] B.T. Doshi (1990). Single server queues with vacations. In: Stochastic Analysis of Computer and Communication Systems. H. Takagi (ed.). North-Holland Publ. Co., Amsterdam, pp. 217-265.

[5] O. Kella (1998). An exhaustive Lévy storage process with intermittent output. Stochastic Models 14, 979-992.

[6] O. Kella, O.J. Boxma and M. Mandjes (2007). On correlated busy periods and service interruptions. In preparation.

[7] K. Sato (1999). Lévy processes and infinitely divisible distributions. Cambridge University Press, Cambridge, UK.

[8] H. Takagi (1991). Queueing Analysis. Vol. 1: Vacation and Priority Systems. NorthHolland Publ. Co., Amsterdam. 